\title{
Farinha do albedo do maracujá (Passiflora edulis Sims) para o enriquecimento de produtos cárneos: Uma revisão
}

\author{
Passion fruit albedo flour (Passiflora edulis Sims) for meat product enrichment: A review \\ Harina de albedo de maracuyá (Passiflora edulis Sims) para el enriquecimiento de productos \\ cárnicos: Una revisión
}

Recebido: 27/06/2021 | Revisado: 04/07/2021 | Aceito: 07/07/2021 | Publicado: 09/07/2021

Kerliana Paula Amorim de Moura

ORCID: https://orcid.org/0000-0003-2253-8349 Instituto Federal de Educação, Ciência e Tecnologia do Ceará, Brasil E-mail: kerligryffindor@gmail.com

Ana Paula Ferreira de Almeida

ORCID: https://orcid.org/0000-0002-3573-2987 Instituto Federal de Educação, Ciência e Tecnologia do Ceará, Brasil E-mail: ana.paula.ferreira.almeida123@gmail.com

Ana Thaís Campos de Oliveira

ORCID: https://orcid.org/0000-0002-8996-6092 Instituto Federal de Educação, Ciência e Tecnologia do Ceará, Brasil

E-mail: euthaiscampos.alimentos@gmail.com

Verônica Freire Queiroz

ORCID: https://orcid.org/0000-0002-3404-6635 Instituto Federal de Educação, Ciência e Tecnologia do Ceará, Brasil E-mail: vevequeiroz53@gmail.com

Kelvi Wilson Evaristo Miranda

ORCID: https://orcid.org/0000-0002-5932-4996

Universidade Federal do Ceará, Brasil E-mail: kelviwilmiranda@gmail.com

Luana Guabiraba Mendes

ORCID: https://orcid.org/0000-0001-8184-8588

Universidade Federal do Ceará, Brasil

E-mail: luanagmendes@gmail.com

Antonia Lucivania de Sousa Monte

ORCID: https://orcid.org/0000-0003-2504-5136

Instituto Federal de Educação, Ciência e Tecnologia do Ceará, Brasil

E-mail: lucivania@ifce.edu.br

\begin{abstract}
Resumo
Produtos cárneos apresentam altos percentuais de gordura, principalmente saturada. Estudos mostram a viabilidade da substituição da gordura por outros tipos de ingredientes, afim de melhorar o conteúdo nutricional. O albedo do maracujá é visto como potencial substituto da gordura, por possuir elevado nível de fibras, proteína e minerais. Além disso, é rico em pectina, um polissacarídeo formado por ácido galacturônico. O presente estudo objetivou identificar na bibliografia científica, a relevância funcional da farinha do albedo do maracujá no enriquecimento em produtos cárneos. A pesquisa trata de uma Revisão Narrativa, a partir de publicações nas seguintes bases científicos: CAPES, Pubmed, ScienceDirect e Google Acadêmico. A pesquisa consistiu no uso dos seguintes descritores e operadores: "sausage" OR "meat products" AND "passion fruit albedo" na língua inglesa, nos anos 2012 a 2021. O consumo de farinha do albedo de maracujá está associado a vários benefícios a saúde, como o retardamento do esvaziamento gástrico e do trânsito intestinal, aumento da sensação de saciedade, diminuição dos níveis de glicose e colesterol total sanguíneo. A incorporação da farinha do albedo de maracujá na porcentagem de $2,5 \%$ pode provocar alterações nas características físico-químicas e tecnológicas de produtos cárneos. Sendo assim, o seu uso contribui para formulações com alto valor nutricional no produto, reduz os impactos ambientais e agrega valor a partes sólidas do maracujá. Contudo, mais estudos são necessários na área de desenvolvimento de produtos alimentícios e mais caracterizações sobre os benefícios desse enriquecimento e interações bioquímicos com a matriz alimentícia, para posterior comercialização.
\end{abstract}

Palavras-chave: Gordura saturada; Pectina; Fibra dietética; Funcionalidade; Fruto tropical.

\section{Abstract}

Meat products have high percentages of fat, especially saturated. Studies show the feasibility of replacing fat with other types of ingredients in order to improve the nutritional content. Passion fruit albedo is seen as a potential fat substitute, as it has a high level of fiber, protein, and minerals. Furthermore, it is rich in pectin, a polysaccharide formed by 
galacturonic acid. The present study aimed to identify, in the scientific literature, the functional relevance of the passion fruit albedo flour in the enrichment of meat products. The research is a Narrative Review, based on publications in the following scientific databases: CAPES, Pubmed, ScienceDirect, and Academic Google. The research consisted of the use of the following descriptors and operators: "sausage" OR "meat products" AND "passion fruit albedo" in English, in the years 2012 to 2021 . The consumption of passion fruit albedo flour is associated with several health benefits, such as delayed gastric emptying and intestinal transit, increased feeling of satiety, decreased levels of glucose, and total blood cholesterol. The incorporation of passion fruit albedo flour in the percentage of $2.5 \%$ can cause changes in the physicochemical and technological characteristics of meat products. Therefore, its use contributes to formulations with high nutritional value in the product, reduces environmental impacts, and adds value to solid parts of passion fruit. However, more studies are needed in the area of food product development and more characterizations about the benefits of this enrichment and biochemical interactions with the food matrix, for further commercialization.

Keywords: Saturated fat; Pectin; Dietary fiber; Functionality; Tropical fruit.

\section{Resumen}

Los productos cárnicos tienen altos porcentajes de grasas, especialmente saturadas. Los estudios demuestran la viabilidad de sustituir la grasa por otro tipo de ingredientes para mejorar el contenido nutricional. El albedo de la maracuyá se considera un posible sustituto de la grasa, ya que tiene un alto nivel de fibra, proteínas y minerales. Además, es rico en pectina, un polisacárido formado por ácido galacturónico. El presente estudio tuvo como objetivo identificar, en la literatura científica, la relevancia funcional de la harina de albedo de maracuyá en el enriquecimiento de productos cárnicos. La investigación es una revisión narrativa, basada en publicaciones en las siguientes bases de datos científicas: CAPES, Pubmed, ScienceDirect y Academic Google. La investigación consistió en el uso de los siguientes descriptores y operadores: "sausage” OR “meat products” AND "passion fruit albedo" en inglés, en los años 2012 a 2021. El consumo de harina de albedo de maracuyá se asocia con varios beneficios para la salud, como retraso en el vaciamiento gástrico y el tránsito intestinal, aumento de la sensación de saciedad, disminución de los niveles de glucosa y colesterol total en sangre. La incorporación de harina de albedo de maracuyá en un porcentaje del 2,5\% puede provocar cambios en las características fisicoquímicas y tecnológicas de los productos cárnicos. Por tanto, su uso contribuye a formulaciones con alto valor nutricional en el producto, reduce los impactos ambientales y agrega valor a las partes sólidas de la maracuyá. Sin embargo, se necesitan más estudios en el área de desarrollo de productos alimenticios y más caracterizaciones sobre los beneficios de este enriquecimiento y las interacciones bioquímicas con la matriz alimentaria, para su posterior comercialización.

Palabras clave: Grasa saturada; Pectina; Fibra dietética; Funcionalidad; Fruta tropical.

\section{Introdução}

Com a vida cada vez mais agitada e menos tempo disponível para o preparo de uma alimentação saudável, os consumidores estão procurando por praticidade, qualidade (nutricional e sensorial) e segurança alimentar nos produtos, os chamados fast food (alimentação rápida) (Souza et al., 2021). Além disso, buscam por conveniência, por alimentos que contribuam para um estilo de vida mais saudável (healthy lifestyle) (Hartmann et al., 2020). Carnes e produtos cárneos se enquadram "quase" que perfeitamente nestas exigências, pois além de serem extremamente práticos, são componentes importantes para uma dieta rica em proteínas, ácidos graxos, vitaminas e minerais (Das et al., 2020; Hartmann et al., 2020).

No entanto, por que "quase" perfeitos? Segundo a literatura, os produtos cárneos apresentam baixo teor de fibras, altos percentuais de gordura, principalmente gordura saturada que está relacionada com o aumento do colesterol LDL (low density lipoprotein), e do risco de desenvolver doenças como diabetes, câncer, obesidade e doenças cardiovasculares (Gedikoglu \& Clarke, 2019; Hartmann et al, 2020). No entanto, a gordura é um componente importante para o alimento, pois influência na maciez, suculência e sabor, entre outros atributos (Silva et al., 2020). Além disso, apresenta importância para a constituição da membrana plasmática, devido a presença dos fosfolipídios e colesterol, os primeiros, são responsáveis por preservar a disposição estrutural da mesma, e o segundo por elevar as características da barreira das duas camadas de lipídios e reduzem a movimentação, possibilitando a bicamada ficar com menor fluidez (Biologia, 2021).

A redução do nível de gordura em produtos cárneos pode afetar diretamente na aceitabilidade do alimento e interferir nos aspectos tecnológicos do produto. Entretanto, estudos mostram a viabilidade da substituição da gordura por ingredientes como cana-de-açúcar, óleo de gergelim, farinha de banana verde, fibras de celulose, frutooligossacarídeo, inulina e pectina. Observaando boa aceitabilidade sensorial e melhoria nas características tecnológicas e nutricionais (Hartmann et al. 2020). A 
reformulação de derivados cárneos em uma perspectiva mais nutricional é apresentada como essencial e inovadora, de baixo custo e de impacto positivo ao ambiente com a baixa geração de resíduos sólidos (Silva et al., 2020). A indústria de alimentos é responsável por gerar grandes quantidades de desperdício, estudos tem mostrado que cerca de 1,3 bilhões de toneladas de comida são desperdiçadas mundialmente por ano, desta quantidade cerca de 0,5 bilhões são de frutas e vegetais (Silva, Ortiz, Asquieri \& Damiani, 2020). No maracujá por exemplo, são desprezados aproximadamente $40 \%$ do total do peso do mesmo, a partir de suas cascas e sementes que são obtidos do processamento do corte e remoção da fruta para aquisição do suco (Oliveira et al., 2012).

Maracujá é um nome genérico dado para diversas espécies do gênero Passiflora, estima-se que existam mais de 500 espécies. A maioria delas é encontrada em países tropicais, com mais de 100 espécies encontradas no Brasil, fazendo com que o maracujá seja parte da biodiversidade brasileira. Inúmeras espécies estão cresecendo comercialmente, gerando empregos nas cidades e no campo, apresentando alto valor econômico e social, tornando o seu cultivo uma ótima alternativa para a agricultura familiar (Faleiro, Junqueira, Junghans, Jesus, Miranda, Otoni, 2019). A espécie mais explorada comercialmente é a Passiflora edulis Sims, que inclui tanto a variedade amarela quanto a roxa, ambas são usadas majoritariamente na indústria de alimentos para produzir sucos e doces, gerando uma enorme quantidade de pele, bagaço, sementes e mucilagem (Voulo et al., 2020; K. Santos et al., 2021; E. Santos et al., 2021). A pele é constituída por flavedo (pigmentação) e albedo (parte branca) (Silva et. al., 2019), que representa cerca de 50,7\% do peso total da fruta madura e cerca de 79,2\% do peso total da fruta verde (Talma, Regis, Ferreira, Mellinger-Silva \& Resende, 2019). O restante representa o suco (+/-26,78\%) e as sementes $(+/-3,59 \%)$ (Silva et. al., 2019).

Esses resíduos do maracujá, podem ser matérias primas de coprodutos de potencial tecnológico a ser explorado na área de desenvolvimento de novos produtos alimentícios. Através da formação de componentes com alto valor agregado para uso para agente de substituição, melhoradores tecnológicos no âmbito nutricional e/ou sensorial, além de promover uma redução no impacto da geração de resíduos sólidos ao meio ambiente (Talma et. al., 2019; K. Santos et al., 2021).

O albedo do maracujá apresenta alto teor de fibras, proteína e minerais. Além disso, possui de 2,0\% a 3,0\%, em base úmida de pectina, um polissacarídeo complexo formado por ácido galacturônico, podendo atuar com fibra solúvel que auxilia na redução dos níveis de glicose no sangue, sendo também fonte de vitamina B3 (niacina), ferro, cálcio e fósforo, possui também propriedades gelificantes que é de grande interesse industrial, se torrnando uma alternativa de espessante natural para a indústria (Embrapa, 2021; Oliveira, Andrade, Damasceno, Azevêdo \& Cruz, 2019).

Nesse sentido, o presente estudo teve por objetivo identificar na bibliografia científica a relevância da farinha do albedo do maracujá (Passiflora edulis Sims) na funcionalidade sobre produtos de origem cárnea.

\section{Metodologia}

Para detectar as essenciais peculiaridades da importância da farinha do albedo do maracujá (Passiflora edulis Sims) e a sua funcionalidade no enriquecimento em produtos cárneos, foi realizada uma pesquisa de Revisão Narrativa de Literatura sobre a situação da arte temática selecionada, sendo designada por não apresentar um procedimento metodológico de elevada rigidez científica. Entretanto, possui fundamental relevância para o alcance da compreensão sobre o conteúdo estabelecido, apontando atuais cenários de maior e/ou menor visibilidade na literatura definida (Toledo \& Rodrigues, 2017).

A busca bibliográfica das publicações científicas foi alcançada a partir das seguintes bases de dados eletrônicos: CAPES, Pubmed, ScienceDirect e Google Acadêmico. A mesma foi realizada em maio de 2021, utilizando os descritores e operadores apenas na língua inglesa: "sausage" OR "meat products" AND "passion fruit albedo". Como critérios de inclusão foram estabelecidos, artigos científicos que abordassem o assunto da referida revisão e que fossem publicados nos últimos dez anos 
(2012-2021). E no que diz respeito aos critérios de exclusão, estabeleceu-se: produções duplicadas, teses, dissertações e monografias e produções que diversas que não abordaram a aplicação em produtos de origem cárnea.

Para acessar a publicação, foram usados os seguintes meios: link disponibilizado diretamente nas bases de dados, procura no portal do periódico em que o artigo foi publicado. Depois de selecionar as publicações, foi efetuada a Análise de Conteúdo em três etapas, a saber: a primeira permitiu um olhar genérico dos assuntos a partir de leitura; na segunda etapa, foi realizada a caracterização das referências dos autores e averiguação sucinta dos textos. No final, foi executada a interpretação dos resultados de acordo com a fundamentação dos autores (Wahlbrink, Rempel, Moreschi, \& Rodrigues, 2016).

\section{Resultados e Discussão}

O referido estudo proveniente de uma Revisão Narrativa de Literatura, encontra-se fragmentado em 02 tópicos, sendo exteriorizados a seguir.

\section{Farinha do albedo do maracujá e seus benefícios na indústria alimentícia}

O albedo do maracujá é um ingrediente de interesse para a indústria alimentícia, devido ao seu alto teor de fibra dietética e o seu equilíbrio entre fibra solúvel e insolúvel, no qual tem efeitos benéficos a saúde humana. Além disso, apresenta elevada capacidade de emulsificação, capacidade de inchamento e a sua solubilidade facilita o seu uso como ingrediente em alimentos funcionais. Este material pode vir a promover a redução do teor de gordura, problemas de sinérese e modificação da textura e viscosidade do produtos de origem animal, como os embutidos (Molina-Hernández, Martínez-Correa \& Andrade-Mahecha, 2019).

Segundo a literatura, o uso do albedo do maracujá pode ser na forma de farinha. Dessa forma, facilita o uso pela indústria de alimentos, devido a flexibilização e versatilidade em diferentes tipos de produtos alimentícios cárneos. Talma et al. (2019), observaram que o valor nutricional e tecnológico da farinha de albedo é devido o conteúdo de pectina presente, o que confere propriedades reológicas e funcionais, como de agente estabilizante, espessante e gelificante no processamento de alimentos. Além disso, foi identificada como alternativa para a área de polímeros naturais (biopolímeros) para aplicação comercial, podendo ser utilizada como matrizes biopoliméricas na confecção de embalagens ou agentes melhoradores no processamento de embalagens sintéticas (Melo, Aouada \& Moura, 2017; Pateiro et al., 2021).

O consumo de farinha do albedo de maracujá está associado à vários benefícios à saúde, como o retardamento do esvaziamento gástrico e do trânsito intestinal, aumentando assim a sensação de saciedade, diminuição nos níveis de glicose e colesterol no sangue e auxiliando no controle de doenças, como a obesidade por possuir capacidade de reduzir o LDL e aumentar o HDL. Além de contribuir também no controle de doenças crônicas como câncer ou diabetes (Silva et al., 2019).

Segundo Silva et al. (2019), as farinhas do albedo do maracujá apresentaram baixo teor de pectina, não contendo poder gelificante. Os autores observaram que esse comportamento tenha sido promovido pela temperatura de secagem aplicada na farinha $\left(80^{\circ} \mathrm{C}\right)$, tendo em vista que uma farinha seca à $60^{\circ} \mathrm{C}$ apresentou 6,98 e $13,54 \%$ de pectina nos dois lotes analisados. As altas temperaturas diminuem o teor de pectina das farinhas do albedo do maracujá, a degradando. Portanto, o seu foco está no enriquecimento e na melhoria dos produtos alimentícios, com o seu principal destaque no seu alto teor de fibras. De acordo com Macêdo, Bezerra e Landim (2019) o conteúdo de fibras da farinha do albedo do maracujá foi de aproximadamente 41\%, sendo este um nível extremamente alto, deixando evidente a sua contribuição para o melhoramento de alimentos, principalmente aqueles com um teor baixo de fibras. 


\section{Enriquecimento de produtos cárneos com agentes funcionais da farinha do albedo do maracujá}

O processo de fermentação das fibras solúveis proporciona a formação de ácidos graxos de cadeia curta, gases e outros metabólitos secundários que induzem o crescimento de bactérias benéficas. Já as fibras insolúveis, tem uma alta capacidade de retenção de água, porém possuem uma capacidade mínima ou não de serem fermentadas. Com isso, há o aumento no volume do bolo fecal e melhoramento no trânsito intestinal, estando também associados à redução das taxas de resistência a insulina e ao risco de desenvolvimento de diabetes tipo 2. Além disso, colaboram com a regularização dos movimentos intestinais, prevenindo problemas relacionados ao intestino, protegendo contra infecções virais respiratórias, desenvolvimento de certos tipos de câncer e proporcionando aumento na imunidade (Bernaud \& Rodrigues, 2013; Miranda \& Schmiele, 2020; Das et al., 2020).

Fibras dietéticas solúveis tem a habilidade de formar gel, elevar a viscosidade e propriedades emulsificantes, devido a sua capacidade de hidratação (Mehta et al., 2019) a pectina é um exemplo de fibra solúvel (K. Santos et al., 2021). As principais vantagens relacionadas ao consumo de fibras solúveis são a diminuição índice glicêmico e do risco de doenças cardiovasculares, devido a resistência à digestão, mas sendo facilmente fermentadas por bactéria do cólon em comparação com as fibras insolúveis (Talma et. al., 2019).

Devido as características tecnológicas e funcionais, as fibras alimentícias vêm sendo utilizadas como ingrediente enriquecedores em diversos produtos cárneos. Tais aplicações visam a produção de alimentos mais saudáveis, com características funcionais para o consumidor. Além disso, a adição de fibras colabora para a modificação das características físico-químicas dos produtos cárneos como na capacidade de retenção de água (CRA), na capacidade da retenção de gordura (CRG) e no perfil de textura (Santos, Sousa, Almeida, Gusmão \& Gusmão, 2019). Isso melhora a estabilidade de emulsão, a viscosidade, as características reológicas e os atributos sensoriais dos produtos cárneos (Das et al., 2020).

Segundo Santos et al. (2021), o albedo do maracujá pertence ao grupo de alimentos prebióticos, que são definidos como frutas e vegetais não digeríveis. No entanto, fibras vegetais não são geralmente adicionadas aos produtos cárneos, devido as alterações indesejáveis que podem causar nas características intrínsecas dos produtos e na interação entre os ingredientes. Sendo assim, um grande desafio aos pesquisadores do setor de P\&D (Pesquisa e Desenvolvimento) da indústria, é encontrar uma variação aceitável para aplicação das fibras alimentícias nos diferentes tipos de produtos cárneos.

A incorporação da farinha do albedo de maracujá na porcentagem de 2,5\% provocou alterações nas características físicoquímicas e tecnológicas dos produtos cárneos. Melhoras foram observadas na perda de peso por cozimento, na estabilidade de emulsão, na dureza, na mastigabilidade, e aumento do valor nutricional, exaltando a importância do papel das fibras como suporte tecnológico, principalmente na capacidade de emulsão, auxiliando na absorção de água e gordura (Santos et al., 2021; LópezVargas et al., 2014). Os produtos cárneos enriquecidos com a farinha do albedo do maracujá, se mostraram isentos de microrganismos e foram aceitos sensorialmente, se apresentando aptos para o consumo humano (Santos et al., 2021; Selani et al., 2015).

\section{Conclusão}

O uso da farinha do albedo do maracujá (Passiflora edulis Sims) se mostrou viável em diferentes trabalhos apresentados na literatura científica. A aplicação tecnológica da farinha pode contribuir para diferentes formulações de produto distintos, como hambúrgueres de frango e carne enlatada, agregando alto valor nutricional, reduzindo os impactos ambientais causados pelo o desperdício das partes sólidas do maracujá, as quais tiveram agregação de valor. Contudo, é observado pouca abordagem e aprofundamento de estudos científicos utilizando a farinha do albedo de maracujá em produtos cárneos. Dessa forma, sugere-se para estudos científicos futuros o desenvolvimento de novos produtos alimentícios, com a adição da farinha, como por exemplo, salsicha, mortadela, hambúrgueres (carne, porco, mistos), empanados, entre outros. Avaliando os benefícios nutricionais dos produtos cárneos enriquecidos com esse coproduto alimentício, encontrando a concentração ideal e aplicando em escala 
industrial com avaliação do comportamento do consumidor ao ser deparado com um produto sem gordura, através de testes sensoriais mercadológicos (atitude de compra) para avaliação do nível de aceitabilidade do produto para posterior comercialização.

\section{Agradecimentos}

Ao Programa Institucional de Bolsas de Iniciação Científica (PIBIC), ao Programa Institucional de Bolsas de Iniciação em Desenvolvimento Tecnológico e Inovação (PIBITI), a Coordenação de Aperfeiçoamento de Pessoal de Nível Superior (CAPES) e a Fundação Cearense de Apoio e Desenvolvimento Científico e Tecnológico (FUNCAP) pelo apoio financeiro e suporte de bolsas para as discentes do IFCE- campus Limoeiro do Norte.

\section{Referências}

Biologia, Só. (2021). Membrana plasmática. https://www.sobiologia.com.br/conteudos/Citologia/cito5.php

Bernaud, F. S. R. \& Rodrigues, T. C. (2013). Fibra alimentar - Ingestão adequada e efeitos sobre a saúde do metabolismo. Arq Bras Endocrinol Metab., 57(6), 397-405. https://www.scielo.br/j/abem/a/PZdwfM5xZKG8BmB9YH59crf/?lang=pt\&format=pdf.

Das, A. K., Nanda, P. K., Madane, P., Biwas, S., Das, A., Zhang, W. \& Lorenzo, J. M. (2020). A comprehensive review on antioxidant dietary fibre enriched meat-based functional foods. Food Science \& Technology, 99, 323-336.

Embrapa (2021). Albedo de maracujá amarelo: propriedades físicas e funcionais. https://www.embrapa.br/busca-de-publicacoes/-/publicacao/1021468/albedode-maracuja-amarelo-propriedades-fisicas-e-funcionais.

Faleiro, F. G., Junqueira, N. T. V, Junghans, T. G., Jesus, O. N. de, Miranda, D. \& Ontoni, W. G. (2019). Advances in passion fruit (Passiflora spp.) propagation. Revista Brasileira de Fruticultura, 41(2), e-155. https://doi.org/10.1590/0100-29452019155.

Gedikoglu, A. \& Clarke, A.D. (2019). Quality attributes of citrus fiber added ground beef and consumer acceptance of citrus fiber added Turkish meatballs. Food and Health, 5, 205-214. 19 jun. 2019. https://doi.org/10.3153/FH19022.

Hartmann, G., Texeira, F., Soares. J. M., Silva, K. A. da, Schwarz, K., Schiessel, D. L. \& Novello, D. (2020). Effect of fat replacement by fructooligosaccharide in hamburger: physicochemical, technological and sensorial analysis. International Journal for Innovation Education And Research, 8(3), 15-25. http://dx.doi.org/10.31686/ijier.vol8.iss3.2128.

López-vargas, J. H., Fernández-lópez, J., Pérez-álvarez, J. Á. \& Viuda-martos, M. (2014). Quality characteristics of pork burger added with albedo-fiber powder obtained from yellow passion fruit (Passiflora edulis var. flavicarpa) co-products. Meat Science, 97(2), 270-276. http://dx.doi.org/10.1016/j.meatsci.2014.02.010.

Macêdo, J. C. B., Bezerra, K. C. B. \& Landim, L. A. dos S. R. (2019). Desenvolvimento da farinha do albedo de maracujá amarelo. Nutrição Brasil, 18(3), 151156. https://doi.org/10.33233/nb.v18i3.3523.

Melo, P. T. S., Aouada, F. A. \& Moura, M. R. (2017). Fabricação de filmes bionanocompósitos à base de pectina e polpa de cacau com potencial uso como embalagem para alimentos. Química Nova, 40(3), 247-251. https://doi.org/10.21577/0100-4042.20160188.

Mehta, N., Chatli, M. K., Kumar, P., Malav, O. P., Verma, A. K., Kumar, Y. \& Kumar, D. (2019). Development of dietary fiber-rich meat products: Technological advancements and functional significance. Bioactive Molecules in Food, 763-795. https://doi.org/10.1007/978-3-319-78030-69.

Miranda, A. V. S. \& Schmiele, M. (2020). Non-digestible carbohydrates as an alternative to improve the technological and nutritional quality of meat products and potential application in fish burgers. Research, Society and Development, 9(11), 1-47. https://doi.org/10.33448/rsd-v9i11.10490.

Molina-hernández, J. B., Martínez-correa, H. A. \& Andrade-mahecha, M. M. (2019). Potencial agroindustrial del epicarpio de maracuyá como ingrediente alimenticio activo. Información Tecnológica, 30(2), 245-256. http://dx.doi.org/10.4067/S0718-07642019000200245.

Oliveira, F. A. de, Andrade, A. B., Damasceno, W. R. P., Azevêdo, R. dos R. \& Cruz, R. S. (2019). Preparation of panification products using passion fruit in replacement of wheat flour. $J B T H, 2(3), 99-104$. https://doi.org/10.34178/jbth.v2i3.78.

Oliveira, L. C. de., Santos, J.A. B., Narain, N., Fontes, A. dos S., Campos, R.S.S. \& Souza, T. L.de. (2012). Caracterização e extração de compostos voláteis de resíduos do processamento de maracujá (Passiflora edulis Sims f. flavicarpa degener). Ciência Rural, 42(12), 2280-2287. http://dx.doi.org/10.1590/s010384782012005000103

Pateiro, M., Gómez, B., Munekata, P. E. S., Barba, F.J., Putnik, P., Kovacevic, D. B. \& Lorenzo, J. M. (2021). Nanoencapsulation of Promising Bioactive Compounds to Improve Their Absorption, Stability, Functionality and the Appearance of the Final Food Products, Molecules, 26(1547), 1-26. https://doi.org/10.3390/molecules26061547.

Santos, E. dos A., Ribeiro, A. E. C., Barcellos, T. T., Monteiro, M. L. G., Mársico, E. T., Caliari, M. \& Júnior, M. S. S. (2021). Exploitation of byproducts from the passion fruit juice and tilapia filleting industries to obtain a functional meat product. Food Bioscience, 41,101084. https://doi.org/10.1016/j.fbio.2021.101084. 
Santos, K. de L., Sousa, F. M. de; Almeida, R. D. de, Gusmão, R. P. de \& Gusmão, T. A. S. (2019). Replacement of Fat by Natural Fibers in Chicken Burgers with Reduced Sodium Content. The Open Food Science Journal, 11(1), 1-8. http://dx.doi.org/10.2174/1874256401911010001.

Santos, K. L., Alves, C. A. N., Sousa, F. M., Gusmão, T. A., Filho, E. G. A. \& Vasconcelos, L. B. (2021). Chemometrics applied to physical, physicochemical and sensorial attributes of chicken hamburgers blended with green banana and passion fruit epicarp biomasses. International Journal of Gastronomy and Food Science, 24, 100337. https://doi.org/10.1016/j.ijgfs.2021.100337.

Selani, M. M., Margiotta, G. B.; Piedade, S. M. de S., Contreras-Castillo, C. J. \& Canniatt-Brazaca, S. G. (2015). Physicochemical, Sensory and Cooking Properties of Low Fat Beef Burgers with Addition of Fruit Byproducts and Canola Oil. IPCBEE, 81(11), 58-65.

Silva, E. C. O., Silva, W. P. Da, Gomes, J. P., Silva, C. D. P. da S. E, Souto, L. M. de \& Costa, Z. R. T. (2019). Physico-chemical characteristics of passion fruit flour under removal of flavedo and of maceration. Revista Brasileira de Engenharia Agrícola e Ambiental, 23(11), 869-875. https://doi.org/10.1590/18071929/agriambi.v23n11p869-875.

Silva, J. S., Ortiz, D. W., Asquieri, E. R. \& Damiani, C. (2020) Physicochemical and technological evaluation of flours made from fruit co-products for use in food products. Research, Society and Development, 9(3), e192932742. 10.33448/rsd-v9i3.2742.

Silva, L. B.F., Miranda, C. N., Santos, M. dos, Pereira, P. A. P., Cunha, L. R., Vieira, S. M. \& Gandra, K. M. B. (2020). Farinha de albedo de laranja como substituto de gordura em hambúrgueres bovinos: agregando valor aos subprodutos da citricultura. Pesquisa, Sociedade e Desenvolvimento, 9(10), e1599108298. 10.33448 / rsd-v9i10.8298.

Souza, A. A. de., Barbosa, B. L. N., Rodrigues, J. J. A., Silva, L. M. de T., Figueiredo, N. M., Lima, C. M. G., Nora, F. M. D., Verruck, S., Santos, T. M. dos., Rodrigues, M. A. dos S. \& Caetano, D. (2021). Different contents of fat in pork-based nuggets: a sensorial perspective. Research, Society and Development, 10(1), e32710111410. 10.33448/rsd-v10i1.11410.

Talma, S. V., Regis, S. A., Ferreira, P. R., Mellinger-Silva, C. \& Resende, E. D. de. (2019). Characterization of pericarp fractions of yellow passion fruit: density, yield of flour, color, pectin content and degree of esterification. Food Science and Technology, 39, 683-689. https://doi.org/10.1590/fst.30818.

Toledo, J. A. de \& Rodrigues, M. C. (2017). Teoria da mente em adultos: uma revisão narrativa da literatura. Bol. - Acad. Paul. Psicol, 37(92), 139-156.

Voulo, M. M., Lima, G. C., Batista, Â. G., Carazin, C. B. B., Cintra, D. E., Prado, M. A. \& Júnior, M. R. M. (2020). Passion fruit peel intake decreases inflammatory response and reverts lipid peroxidation and adiposity in diet-induced obese rats. Nutrition Research, 76 , 106-117. https://doi.org/10.1016/j.nutres.2019.08.007.

Wahlbrink, D., Rempel, C., Moreschi, C. \& Rodrigues, J. B. P (2016). Características da hemocromatose: uma revisão narrativa. Saúde (santa maria),1-25, http://dx.doi.org/10.5902/2236583414137. 